\title{
Pedunculated Pulmonary Parenchymal Leiomyoma Associated with Hypertrophic Osteoarthropathy: Case Report
}

\author{
Leonardo Politi ${ }^{*}$, , Camilla E. Comin ${ }^{2}$, Daniel Barale ${ }^{1}$ and Andrea Lopez Pegna ${ }^{3}$ \\ ${ }^{I}$ Department of Medical and Surgical Critical Care, University of Florence, Florence, Italy \\ ${ }^{2}$ Department of Human Pathology and Oncology, University of Florence, Florence, Italy \\ ${ }^{3}$ Pneumology Service, Careggi Hospital, Florence, Italy
}

\begin{abstract}
Hypertrophic osteoarthropathy, a condition of osteitis of the long bones that manifests clinically with clubbing, is often secondary to a thoracic neoplasm, especially lung carcinoma in long-time smokers. We report an unusual case in which hypertrophic osteoarthropathy was the only manifestation of a benign pulmonary parenchymal leiomyoma. A 38year-old non-smoking man had bilateral clubbing of the fingers. Hypertrophic osteoarthropathy was diagnosed when radiography revealed thin lamellar deposits along the metatarsals and phalanges of the hands and similar osteal changes in the feet. Respiratory tests indicated normal lung functioning, but computed tomography revealed a $5 \times 4 \mathrm{~cm} 2$ lobulated mass in the lower left lung which did not take up 18F-FDG on positron emission tomography. Exploratory thoracotomy revealed a pedunculated neoplasm which was removed by standard lobectomy. Histopathological diagnosis indicated the presence of smooth muscle spindle cells but absence of mitotic activity, nuclear atypia or necrosis, leading to a diagnosis of pulmonary parenchymal leiomyoma. Four months after surgery, the patient experienced a noticeable reduction in the finger clubbing. Hypertrophic osteoarthropathy may be the presenting characteristic of benign masses such as pulmonary leiomyoma.
\end{abstract}

Keywords: Lung, peduncolated leiomyoma, hypertrophic ostheoarthropathy.

\section{INTRODUCTION}

Leiomyoma is a benign tumor of the smooth muscle that commonly presents in the female internal genital organs, especially the uterus, and in the digestive tract, especially the esophagus. Localization of leiomyoma in the respiratory tract is infrequent, although various cases have been presented $[1,2]$. Respiratory tract leiomyomata have been classified into three groups - tracheal, bronchial and parenchymal - which differ in clinical manifestation and diagnostic approach 2.

Leiomyoma has, in a few cases, been associated with hypertrophic osteoarthropathy, a condition of bilateral limb osteitis usually affecting the phalanges and distal ends of the long bones and manifesting clinically with clubbing and periostosis. Also called Marie-Bamberger syndrome, this condition is usually secondary to chronic respiratory disease or to pathologies causing arteriovenous shunting, e.g. lung carcinoma, mesothelioma and inflammatory bowel disease [3]. Of the few cases of hypertrophic osteoarthropathy secondary to leiomyoma, the smooth muscle tumor has been localized to the esophagus [4-7], ileum [8] and, in three cases reported in 1958, also in the lung [9]. We report an unusual case of pulmonary parenchymal leiomyoma that presented as hypertrophic osteoarthropathy.

*Address correspondence to this author at the Department of Medical and Surgical Critical Care, Azienda Ospedaliero Universitaria Careggi, Viale Morgagni 85, I-50134 Firenze, Italy; Tel: 00390557947781;

Fax: 00390557947781; E-mail:leonardo.politi@unifi.it

\section{CASE REPORT}

A 38-year-old, non-smoking, male, chef presented in 2008 for worsening of the clubbing of the fingers of both hands; this symptom had first appeared in 2003. The patient had a history of anaphylactic shock at age 14 years. At age 15 years, he suffered allergic asthma triggered by cypress and grass pollen, which resolved with treatment with disodium cromoglycate and salbutamol. In 1999, he underwent surgery of the cruciate ligaments of the right knee and experienced postoperative hemarthrosis due to factor VIII deficiency diagnosed in the postoperative period.

\section{CLINICAL FINDINGS}

Visual inspection of the hands revealed, in addition to the clubbing, watch-crystal nails and hype-remic congestion (Fig. 1). Radiographs of the hands revealed thin lamellar deposits along the metacarpals and proximal, but not distal, phalanges. Similar changes were observed on the metatarsals and phalanges of the feet, despite the absence of toe clubbing (data not shown). These findings suggested a diagnosis of hypertrophic osteoarthropathy (Marie-Bamberger syndrome). However, respiratory testing did not reveal pulmonary dysfunction: the forced vital capacity (FVC) was 4.571 (108\% of predicted) and the forced expiratory volume in 1 second (FEV1) was 4.201 (115\% of predicted). Arterial blood gas testing also gave normal values: partial pressure of oxygen $\left(\mathrm{PaO}_{2}\right), 102 \mathrm{mmHg}$; oxygen saturation $\left(\mathrm{SaO}_{2}\right)$, 97.1\%; and partial pressure of carbon dioxide $\left(\mathrm{PaCO}_{2}\right), 38$ $\mathrm{mmHg}$. Also the others blood exams (e.g. white cells count, red blood cells count etc.) were normal and no history of 


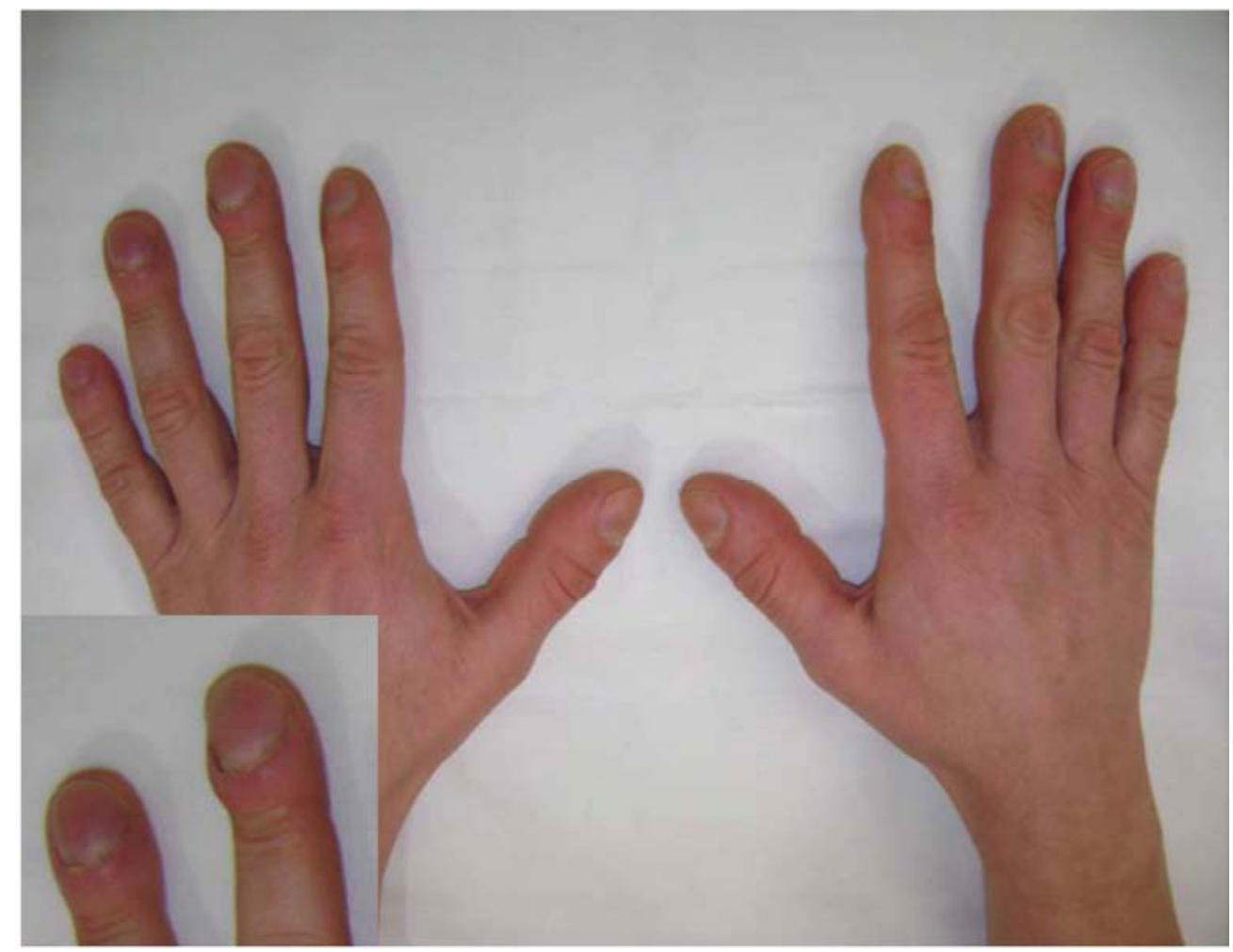

Fig. (1). Hands of a 38-year-old man who presented with a worsening of finger clubbing, first noticed 5 years earlier. a Photograph shows watch-crystal nails and congestive hyperemia of the finger tips.

recurrent viral infections was present so the pathogenetic theory from literature [20] that link immune suppression, Epstein-Barr Virus and hypertrophic osteoarthropathy in children doesn't seems consistent with our case. The serum level of grow hormone was in normal range $(<5 \mathrm{ng} / \mathrm{ml})$ so we we excluded the endocrine pathogenetic theory too [21].

To understand the etiology of the patient's symptoms, we first performed computed tomography (CT) of the chest, which revealed a $5 \times 4 \mathrm{~cm}^{2}$ lobulated mass in the lower lobe of the left lung (Fig. 3), the mass was retrocardiac and paraaortic and adherent to the pericardium and esophagus. Then, positron emission tomography (PET) (Fig. 2) revealed that the lesion did not take up $18 \mathrm{~F}$-fluorodeoxyglucose and the standard uptake value (SUV) was 1.0, excluding a malignant nature.

Considering the anatomical location of the lesion, exploratory thoracotomy was performed. Surgery revealed a lumpy, pedunculated neoplasm with a broad base (Fig. 4). The mass was dissected from the inferior posterior mediastinum and aortic adventitia, which was thickened and pseudofibrotic. Intraoperative frozen section analysis of a tumor specimen indicated the presence of spindle cells, suggesting a smooth muscle tumor, but did not rule out malignancy. Therefore, we performed a standard lobectomy with mediastinal lymph node dissection.

\section{PATHOLOGICAL FINDINGS}

Gross examination of the excised tumor revealed a hard, gray-white, well circumscribed mass measuring $5 \times 2.5 \mathrm{~cm}^{2}$. Histologically, the tumor was composed of orderly,

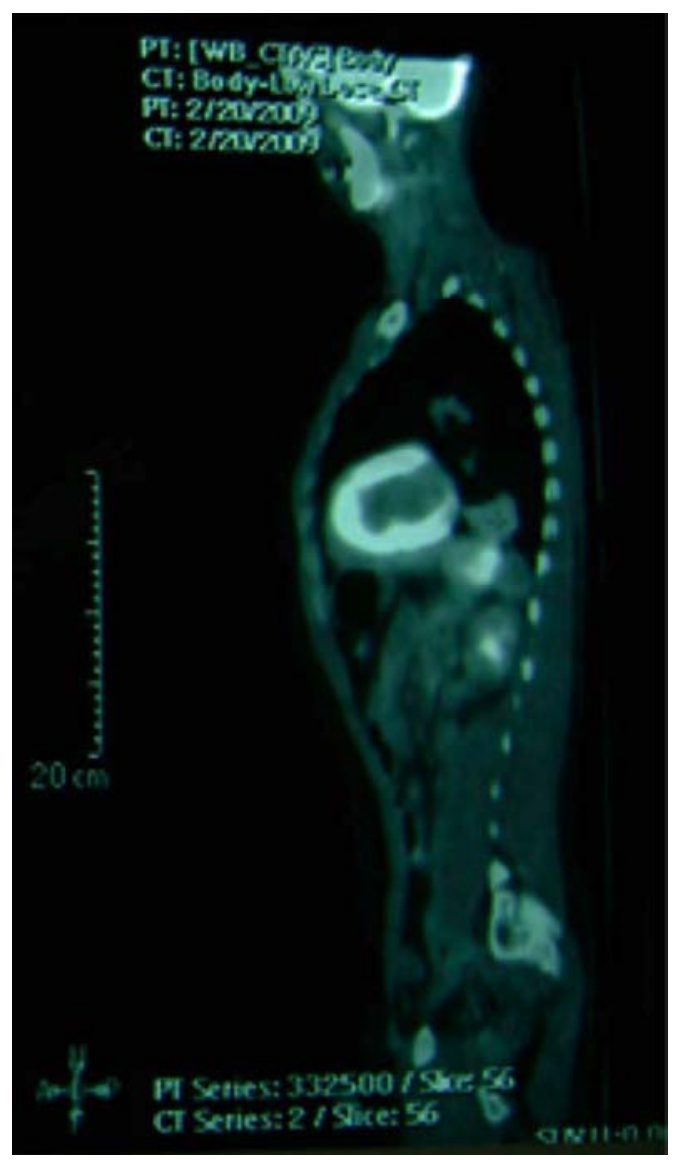

Fig. (2). Sagittal PET scan (left) shows no uptake of 18F-FDG by the pulmonary mass. 


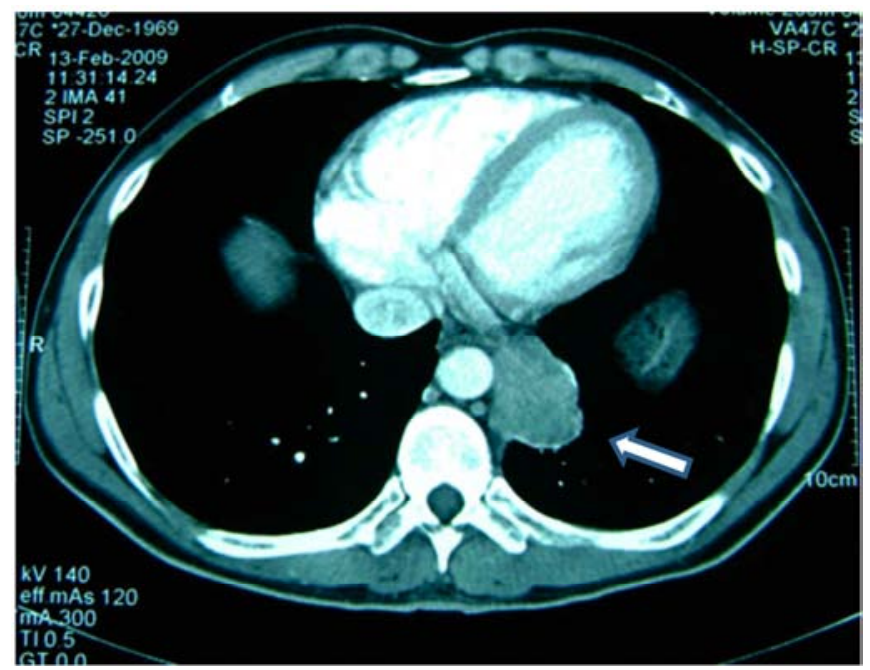

Fig. (3). CT scan shows a $5 \times 4 \mathrm{~cm} 2$ mass (arrow) adherent to the descending thoracic aorta, the lower third of the esophagus and the pericardium.

intersecting fascicles of spindle-shaped cells with eosinophilic cytoplasm and uniform, blunt-ended, cigarshaped nuclei (Fig. 6). Nuclear atypia, necrosis and hemorrhage were absent; no mitotic activity was observed. Immunohistochemical staining showed that the tumor cells were strongly and diffusely positive for smooth muscle actin (Fig. 7), muscle-specific actin, desmin, h-caldesmon and vimentin. Cytokeratins and S-100 protein were negative. Based on the morphologic and immunohistochemical findings, a diagnosis of leiomyoma was established. Surgical margins and lymph nodes were free of disease.

\section{SHORT-TERM FOLLOW-UP}

Four months after the surgery, clinical examination revealed an approximately $80 \%$ reduction in finger clubbing based on profile angle, hyponychial angle, and phalangeal depth ratio (Fig. 5).

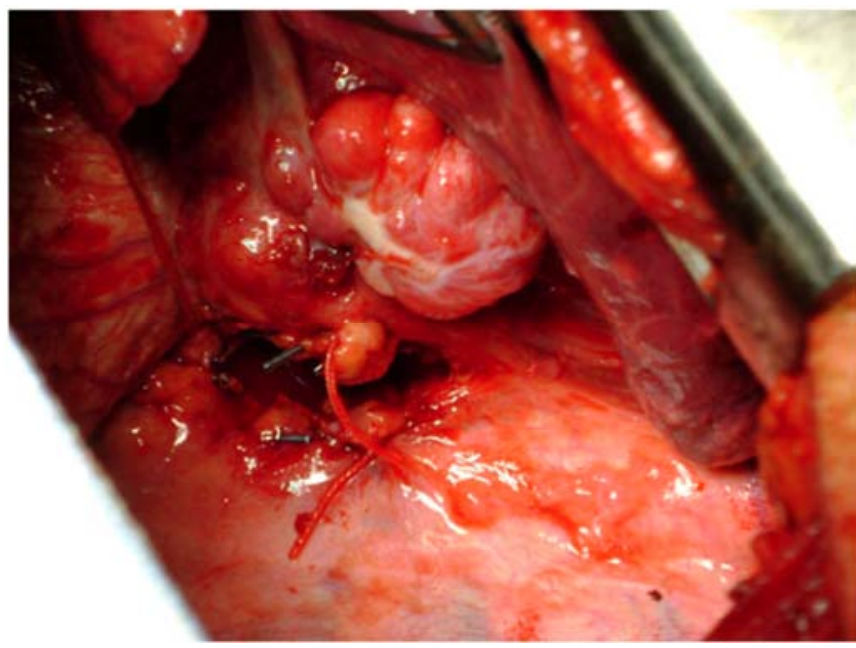

Fig. (4). Intraoperative photograph of the pedunculated mass attached to the left lower pulmonary lobe, aorta and mediastinum.

\section{DISCUSSION}

This rare case illustrates an association between pulmonary parenchymal leiomyoma and hypertrophic osteoarthropathy, which was the only clinical manifestation. A causal relationship is possible, given the improvement in finger clubbing after tumor excision and the fact that the patient had no other known chronic or acute condition that could cause the distal pathology. The association of pulmonary parenchymal leiomyoma with hypertrophic osteoarthropathy has only been described in 3 other cases, in a report published more than 50 years earlier [9]. That report described one pedunculated and one chondromatous tumor, both lobulated, while the third tumor was not resected. In the first two cases, like in this case, finger clubbing resolved after pulmonary resection.

The diagnosis of pulmonary leiomyoma in a 38 -year-old man is fitting with the mean age at presentation of 35-40 years, although cases have been reported at all ages $[1,2]$.

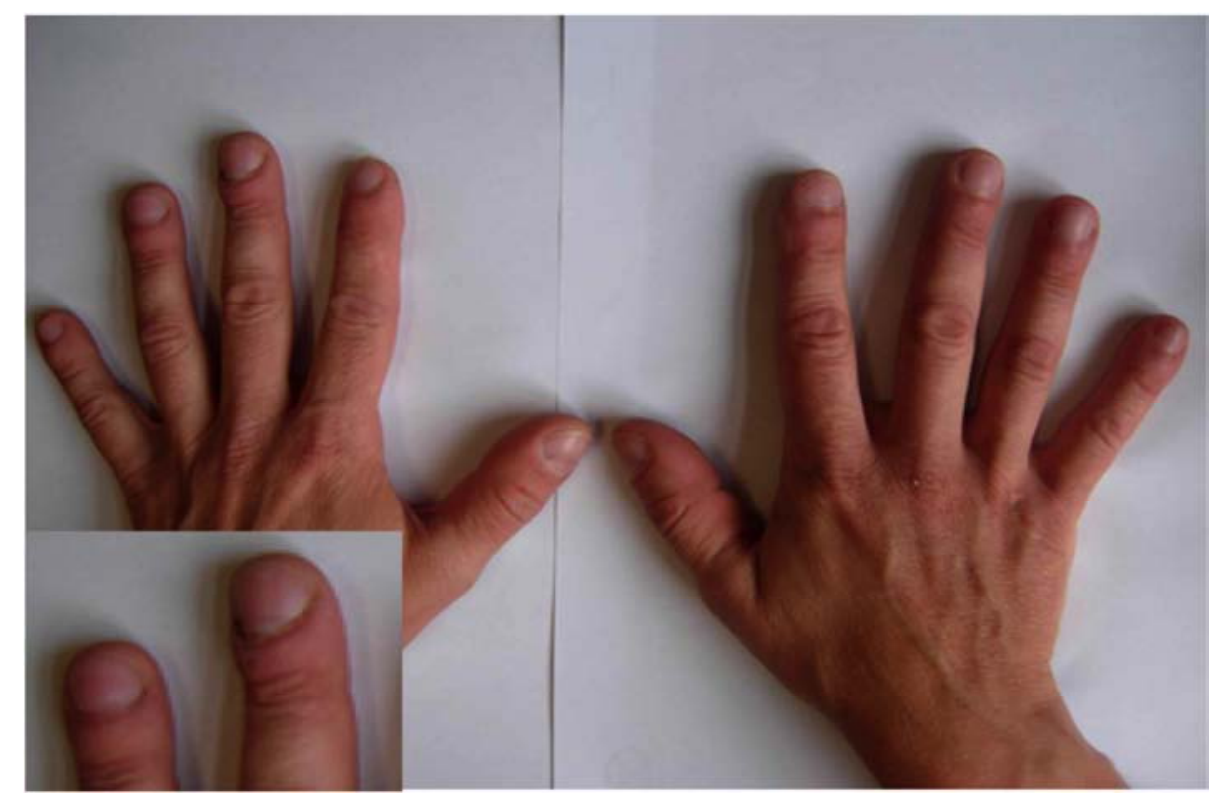

Fig. (5). Photograph of the hands 6 months after excision of a pulmonary leiomyoma reveals substantial reduction in the extent of finger clubbing. 
Localization in the parenchyma is also an usual finding, as about half of all lung sites of leiomyoma are parenchymal, followed by bronchial and then tracheal sites [2]. However, parenchymal localizations are twice as common in women than in men [2]. Recently the literature reports cases of regression of leiomyoma in young women treated with progesteron therapy [22] but in the case of our patient, a male 38 years old, we didn't consider this therapeutic strategy accetable. The pedunculated nature of the lesion is also unusual: although uterine leiomyomata (fibroids) are often pedunculated, only a few cases of pedunculated pulmonary leiomyomata have been reported [10,11], including one associated with hypertrophic osteoarthropathy [9].

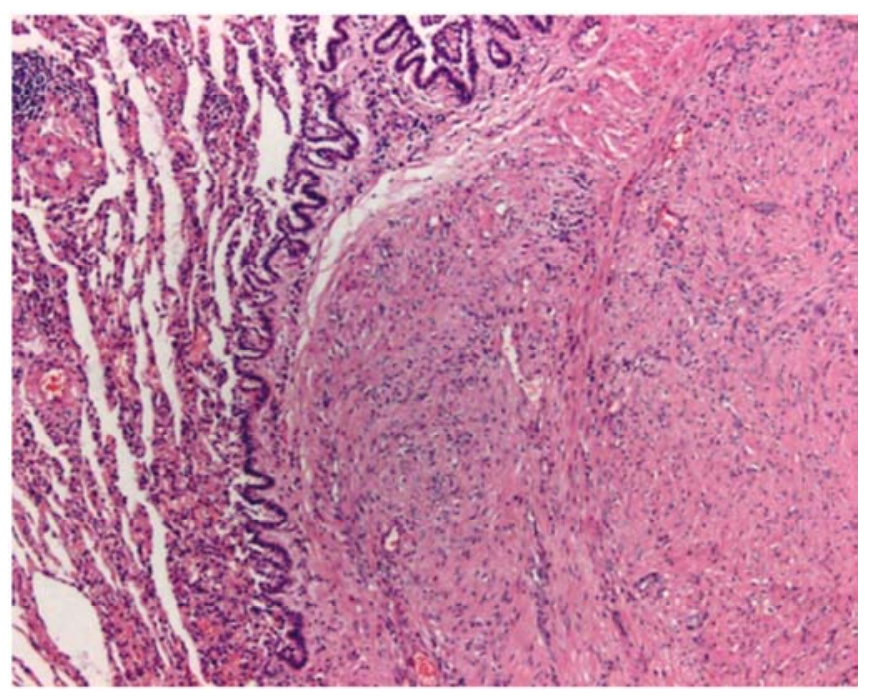

Fig. (6). Histopathological and immunochemical assessment of tumor specimen. a Low-power view showing normal lung parenchyma (left) and leiomyoma (right).

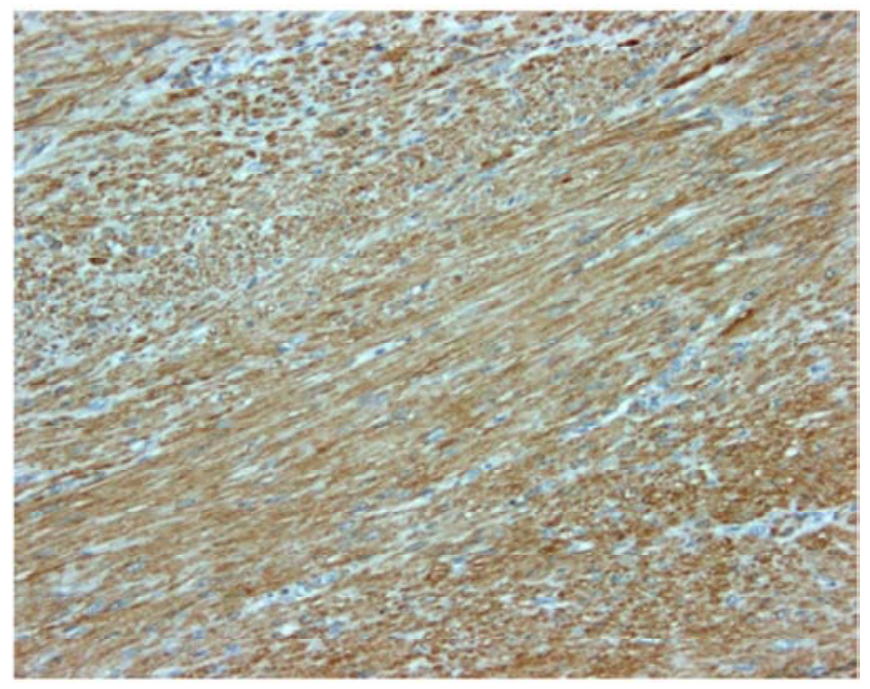

Fig. (7). Fascicles of tumor cells with elongated, blunt-ended nuclei and eosinophilic cytoplasm characteristic of smooth muscle cells.

The ease of diagnosis of pulmonary leiomyoma depends on the anatomical location. Bronchial and tracheal tumors can be examined with endoscopy and biopsied, while parenchymal tumors are difficult to localize and diagnose by non invasive imaging and fine needle aspiration biopsy. In this case, as reported previously [12], the tumor did not take up ${ }^{18} \mathrm{~F}$-FDG due to its low metabolic activity. Moreover, the CT appearance of the lesion is not pathognomonic, due to the lobulated form and the typical absence of calcification and hilar-mediastinal lymphadenopathy [13]. The histopathological diagnosis, using a limited panel of immunohistochemical markers, is usually straightforward, as it was in this case. Difficulties may arise in distinguishing leiomyoma from its malignant counterpart, leiomyosarcoma. The most important differential diagnosis criteria for leiomyoma are the lack of mitotic activity, nuclear atypia and necrosis [14].

Therapy for symptomatic lung leiomyomata usually involves limited resection, since the lesions are benign and usually well delimited [2]. However, extensive resection may be necessary in the presence of irreversible lung damage distal to the tumor, and has been reported in $51 \%$ of cases surveyed in 198 [2]. In the present case, we performed lobectomy because of the intimate relationship of the tumor with the mediastinum, aortic wall and esophagus and because the intra-operative frozen section did not rule out a malignant lesion.

Also for the leiomyoma, as for the other tumors, have been suggested multiple pathogenetic theories to explain the associated syndrome of Pierre Marie and in particular the toxic, the autonomic, the endocrine, the one linked to the pulmonary reflex and the multifactorial one $[9,15]$.

Experimental animal studies [16] conducted by pulmonary arterio-venous anastomoses showed periosteal ossification and arterio-venous fistulas of the limbs. Mauer [17] demonstrated that hypoxia can induce peripheral blood sedimentation responsible for a subperiosteal exudate, while Voegetin and Willie [18] believe that the osteo-articular damages may be due to peripheral neurodegenerative and neurovascular alterations.

Even if in the bronchial carcinomas have not been found pericapillar vascular anastomosis [19], however it is believed that a ischemia of the peripheral nerves of the lung at the level of arterio-venous exchanges would be able to determine a reflex responsible for osteogenesis secondary to arterio-venous exchanges of the limbs. This hypothesis appears more likely even according to Luko and Toth in the cases of leiomyomas with Pierre-Marie-Blomberg syndrome. This theory, always according to the same authors, would be supported by the regression of clubbing in the two cases resected at one year postoperatively, and would be due to the cessation of the reflex stimulation.

\section{CONFLICT OF INTEREST}

The author confirms that this article content has no conflict of interest.

\section{ACKNOWLEDGEMENTS}

Declared none.

\section{REFERENCES}

[1] Vera-Román JM, Sobonya RE, Gomez-Garcia JL et al. Leiomyoma of the lung. Literature review and case report. Cancer 1983; 52: 936-41.

[2] White SH, Ibrahim NB, Forrester-Wood CP, Jeyasingham K. Leiomyomas of the lower respiratory tract. Thorax 1985; 40: 30611 . 
[3] Armstrong DJ, McCausland EM, Wright GD. Hypertrophic pulmonary osteoarthropathy (HPOA) (Pierre Marie-Bamberger syndrome): two cases presenting as acute inflammatory arthritis. Description and review of the literature. Rheumatol Int 2007; 27 : 399-402.

[4] Maekawa H, Tanaka N, Hashimoto N, et al. Esophageal smooth muscle tumor in a 25-year-old woman with congenital malformations. J Gastroenterol 2001; 36(10): 700-3.

[5] Massicot R, Aubert D, Mboyo A et al. Localized esophageal leiomyoma and hypertrophic osteoarthropathy. J Pediatr Surg 1997; 32(4): 646-7.

[6] Kaymakcalan H, Sequeria W, Barretta $\mathrm{T}$ et al. Hypertrophic osteoarthropathy with myogenic tumors of the esophagus. Am J Gastroenterol 1980; 74(1): 17-20.

[7] Ullal SR. Hypertrophic osteoarthropathy and leiomyoma of the esophagus. Am J Surg 1972; 123(3): 356-8.

[8] Ferguson R, Toghill PJ, Bourke J. Reversal of finger clubbing after resection of a leiomyoma of the ileum. Br Med J 1978; 1(6106): 151-2.

[9] Luko G, Toth F. Osteoarthropathy hypertrophiante pneumique (Bamberger-Pierre Marie) with benign lung tumors (leiomyoma). Bruns Beitr Klin Chir 1958; 196(1): 19-31.

[10] Gotti G, Haid MM, Paladini P, et al. Pedunculated pulmonary leiomyoma with large cyst formation. Ann Thorac Surg 1993; 56: 1178-80.

[11] Lajos TZ, Meckstroth CV. Pedunculated leiomyoma of the lung. Report of a case. Dis Chest 1967; 52(1): 114-6.

[12] di Scioscio V, Feraco P, Miglio L, et al. Benign metastasizing leiomyoma of the lung: PET findings. J Thorac Imaging 2009; 24(1): 41-4.
[13] Abramson S, Gilkeson RC, Goldstein JD, et al. Benign metastasizing leiomyoma: clinical, imaging, and pathologic correlation. AJR Am J Roentgenol 2001; 176(6): 1409-13.

[14] Billings SD, Folpe AL, Weiss SW. Do leiomyomas of deep soft tissue exist? An analysis of highly differentiated smooth muscle tumors of deep soft tissue supporting two distinct subtypes. Am J Surg Pathol 2001; 25: 1134-42.

[15] Kozak KR, Milne GL, Morrow JD, Cuiffo BP. Hypertrophic osteoarthropathy pathogenesis: a case highlighting the potential role for cyclo-oxygenase-2-derived prostaglandin E2. Nat Clin Pract Rheumatol 2006; 2: 452-6.

[16] Mendlowitz M, Leslie A: The experimental simulation in the dog of the cyanosis and hypertrophic osteoarthropathy which are associated with congenital heart disease. Am Heart J 1942; 24: 141.

[17] On the Etiology of Clubbing of the Fingers. Edgar F. Mauer Am Heart J 1947; 852-9.

[18] Pulmonary osteoarthropathy and its pathogenesis. Voegtlin R, Willie A. Strasb Med 1955; 6(3): 155-62.

[19] Cudkowicz L, Armstrong JB. Finger clubbing and changes in the bronchial circulation. Br J Tuberc Dis Chest 1953; 47(4): 227-32.

[20] User IR, Dogru D, Talim B, Orhan D, Karnak I. Endobronchial, pulmonary and liver leiomyomata in a child with primary immune deficiency. Eur J Pediatr Surg 2010; 20(6): 423-5.

[21] The relationship between digital clubbing and serum growth hormone level in patients with lung cancer. Yorgancioğlu A, Akin M, Demtray M, Derelt S. Monaldi Arch Chest Dis 1996; 51(3): 185-7.

[22] Taveira-DaSilva AM, Alford CE, Levens ED, Kotz HL, Moss J. Favorable response to antigonadal therapy for a benign metastasizing leiomyoma. Obstet Gynecol. 2012; 119 (2 Pt 2): 43842.

(C) Politi et al.; Licensee Bentham Open.

This is an open access article licensed under the terms of the Creative Commons Attribution Non-Commercial License (http://creativecommons.org/licenses/by$\mathrm{nc} / 3.0 /$ ) which permits unrestricted, non-commercial use, distribution and reproduction in any medium, provided the work is properly cited. 